\title{
Prematuridade e malformações congênitas em recém-nascido: Um relato de caso
}

\author{
Prematurity and congenital diseases in newborn: A case report \\ Precocidad y enfermedades congénitas del recién nacido: Reporte de un caso
}

Recebido: 09/05/2021 | Revisado: 19/05/2021 | Aceito: 25/05/2021 | Publicado: 09/06/2021

\author{
Nathália Menezes Dias \\ ORCID: https://orcid.org/0000-0002-2166-5293 \\ Universidade do Estado do Pará, Brasil \\ E-mail: menezesdiasnathalia@gmail.com \\ Ana Beatriz Capela Cordovil \\ ORCID: https://orcid.org/0000-0002-3080-6553 \\ Universidade do Estado do Pará, Brasil \\ E-mail: anabeatrizcapelac@gmail.com \\ Nayara de Fátima Cardoso Pereira da Silva \\ ORCID: https://orcid.org/0000-0002-5462-3751 \\ Universidade do Estado do Pará, Brasil \\ E-mail: nayaradefatima678@gmail.com \\ Ana Caroline de Oliveira Coutinho \\ ORCID: https://orcid.org/0000-0003-2558-9643 \\ Universidade do Estado do Pará, Brasil \\ E-mail: coutinhoanacaroline@gmail.com \\ Leticia Regina Maia Cordeiro \\ ORCID: https://orcid.org/0000-0003-1972-6832 \\ Universidade do Estado do Pará, Brasil \\ E-mail:enf.leticiarmaia@ hotmail.com \\ Francisco dos Santos Pereira \\ ORCID: https://orcid.org/0000-0003-3127-0954 \\ Universidade Anhanguera, Brasil \\ E-mail: franciscojr30@hotmail.com \\ Lêda Lima da Silva \\ ORCID: https://orcid.org/0000-0002-8729-2290 \\ Universidade do Estado do Pará, Brasil \\ E-mail: ledals500@gmail.com \\ Anderson Bentes de Lima \\ ORCID: https://orcid.org/0000-0002-0534-2654 \\ Universidade do Estado do Pará, Brasil \\ E-mail: andersonbentes@uepa.br
}

\begin{abstract}
Resumo
Objetivo: Relatar um caso de malformação neonatal de três tipos: dedo extranumerário em mão esquerda, genitália ambígua e anus impérvio. Método: O presente estudo trata-se de um relato de caso, com informações obtidas por meio de revisão de ase documental do tipo prontuário. Discursão: Acredita-se que conhecer o perfil das crianças nascidas com malformação congênita seja importante para a assistência, no sentido de oferecer subsídios à melhoria da qualidade da demanda profissional prestada no nascimento de uma criança com malformação congênita. Para que ocorra redução das taxas de morbimortalidade no período neonatal, torna-se necessário conhecer as particularidades regionais de cada serviço, como a frequência das malformações congênitas, a identificação dos fatores de riscos associados, além de valorizar o diagnóstico precoce, prevenções primária e secundária. Logo, vale ressaltar que a existência de algum tipo de malformação congênita tanto no nascimento, como na causa do óbito de crianças é atrelado ao sub-registro, ou seja, ainda existem falhas ao coletar informações acerca deste tipo de ocorrência observada em todas as regiões do Brasil, o que revela a importância de se trabalhar mais fortemente as políticas de promoção e prevenção, direcionada a saúde materno e infantil, para que sejam minimizados o acontecimento de malformações. Conclusão: Dessa forma, a contribuição deste estudo constitui grande relevância para o norteamento na adoção de medidas para o fortalecimento da política do pré-natal com condutas preventivas e delineamento de ações estratégicas no combate aos fatores e causas evitáveis afim de reduzir essas alterações que podem trazer sequelas irreversíveis aos neonatos no seu processo de crescimento e desenvolvimento.
\end{abstract}

Palavras-chave: Doenças e anormalidades congênitas, hereditárias e neonatais; Prematuridade; Enfermagem.

\footnotetext{
Abstract

Objective: To report a case of neonatal malformation of three types: supernumerary finger on the left hand, ambiguous genitalia and impervious anus. Method: The present study is a case report, with information obtained
} 
through the review of documentary ase of the medical record type. Discourse: It is believed that knowing the profile of children born with congenital malformation is important for assistance, in the sense of offering subsidies to improve the quality of the professional demand provided in the birth of a child with congenital malformation. For the reduction of morbidity and mortality rates in the neonatal period, it is necessary to know the regional particularities of each service, such as the frequency of congenital malformations, the identification of associated risk factors, in addition to valuing early diagnosis, primary and secondary preventions. . Therefore, it is worth mentioning that the existence of some type of congenital malformation both at birth and in the cause of the death of children is linked to underreporting, that is, there are still flaws in collecting information about this type of occurrence observed in all regions. from Brazil, which reveals the importance of working more strongly on promotion and prevention policies, aimed at maternal and child health, so that the occurrence of malformations is minimized. Conclusion: Thus, the contribution of this study is of great relevance for guiding the adoption of measures to strengthen the prenatal policy with preventive measures and the design of strategic actions to combat preventable factors and causes in order to reduce these changes that may bring irreversible consequences to neonates in their growth and development process.

Keywords: Congenital, hereditary and neonatal diseases and abnormalities; Prematurity; Nursing.

\section{Resumen}

Objetivo: Informar un caso de malformación neonatal de tres tipos: dedo extranumérico de la mano izquierda, genitales ambiguos y ano impermeable. Método: El presente estudio es un reporte de caso, con información obtenida a través de la revisión de ase documental del tipo de historia clínica. Discurso: Se cree que conocer el perfil de los niños que nacen con malformación congénita es importante para la asistencia, en el sentido de ofrecer subsidios para mejorar la calidad de la demanda profesional brindada en el nacimiento de un niño con malformación congénita. Para la reducción de las tasas de morbimortalidad en el período neonatal, es necesario conocer las particularidades regionales de cada servicio, como la frecuencia de malformaciones congénitas, la identificación de factores de riesgo asociados, además de valorar el diagnóstico precoz, primario y secundario. Prevenciones. Por tanto, cabe mencionar que la existencia de algún tipo de malformación congénita tanto en el nacimiento como en la causa de la muerte de los niños está vinculada a la subregistro, es decir, aún existen fallas en la recolección de información sobre este tipo de ocurrencia observadas en de Brasil, lo que revela la importancia de trabajar con más fuerza en las políticas de promoción y prevención, orientadas a la salud maternoinfantil, para minimizar la ocurrencia de malformaciones. Conclusión: Así, el aporte de este estudio es de gran relevancia para orientar la adopción de medidas de fortalecimiento de la política prenatal con medidas preventivas y el diseño de acciones estratégicas para combatir factores y causas prevenibles con el fin de reducir estos cambios que pueden traer consecuencias irreversibles. a los recién nacidos en su proceso de crecimiento y desarrollo.

Palabras clave: Enfermedades y anomalías congénitas, hereditarias y neonatales; Precocidad; Enfermería.

\section{Introdução}

A malformação congênita é uma condição que pode afetar quase todas as partes do corpo do bebê como coração, cérebro, pés etc., danificando a aparência, o funcionamento do corpo ou ambos e também de anomalia congênita ou doença congênita é uma condição de anormalidade que pode ser de caráter estrutural ou funcional sendo que, pode ocorrer ser identificada durante o concepto, no nascimento ou após alguns dias de vida (ADFP, 2021).

As malformações congênitas são consideradas qualquer alterações de estrutura, função ou metabolismo presentes ao nascer, que resultam em anomalias físicas ou mentais, podendo ou não ser simples ou múltiplas e de maior ou menor importância clínica para a criança acometida (Pereira, 2008).

Segundo a Organização Mundial da Saúde (OMS) em 2016 estimou que 303.000 nascidos vivos vão a óbito devido a malformação congênita o que equivale a um percentual de $7 \%$ do total de nascimentos, durante o primeiro mês de vida (Lima et al., 2019).

Todos os anos, em 6\% dos nascimentos em todo o mundo, cerca de 7,9 milhões de crianças nascem com defeitos congênitos graves e quase 3,2 milhões ficam deficientes para toda a sua vida adulta, o que irá necessitar de acompanhamento para seu crescimento e desenvolvimento. As populações estão expostas aos riscos de desenvolverem malformações congênitas, porém a frequência e o tipo variam de acordo com a raça, a etnia e as condições socioeconômicas (Cardoso et al., 2015).

Para o ministério da saúde esta é uma condição que é considerada a segunda principal causa de morte em recémnascidos e crianças menores de cinco anos nas Américas, pois em primeiro lugar está a prematuridade onde pode ocorrer a associação entre as duas situações. Além disso, a cada 33 bebês nascidos no mundo um irá apresentar defeito congênito, e 
mesmo com algumas anomalias congênitas não serem consideradas fatais, muitas crianças que sobrevivem têm maior risco de apresentarem deficiências em longo prazo, requerendo serviços de saúde e outros serviços de apoio, para melhorar sua qualidade de vida (BVS, 2021).

Conforme Brasil (2018) foi lançado a agenda de prioridades para o ano de 2018 dentre estes está a análise da mortalidade infantil e da qualidade de vida das crianças com anomalias congênitas e o desenvolvimento e validação de metodologia para ampliar a captação precoce e estimar a prevalência de anomalias congênitas por agrupamentos do CID 10.

Verifica-se o alto índice de morbidade e mortalidade neonatal referente as malformações, também, pelo, risco para desenvolvimento de complicações clínicas, com índice de internamentos e gravidade das intercorrências. Vários são os fatores, sejam eles internos ou externos, podem colaborar para o surgimento de um alterações genético, no entanto, a causa é desconhecida na maioria dos casos (Cardoso et al., 2015).

A prematuridade e o baixo-peso têm sido consistentemente associadas às malformações, dentre as malformações, as mais frequentes foram as do sistema nervoso central (principalmente hidrocefalia e meningomielocele), as do sistema osteomuscular (principalmente gastrosquise e onfalocele) e as cardiopatias. A predominância das malformações do sistema nervoso central está de acordo com diversas casuísticas, sobretudo nos países em desenvolvimento (Amorim et al., 2006).

Algumas doenças maternas se configuram como fatores de risco a exemplo da diabetes mellitus, que pode determinar malformação congênita do sistema nervoso central e do aparelho cardiovascular; a hipertensão arterial, o hipotireoidismo, epilepsia que constitui risco moderado para microcefalia e Retardo do Crescimento Intrauterino (CIUR) (Reis \& Ferrari, 2014).

A hospitalização desta criança, consequentemente, o cuidado intensivo implementado aos neonatos em UTIN estão associados à submissão destes a inúmeros de procedimentos sejam eles, como as punções venosas, as sondagens orogástricas e vesicais, as glicemias capilares, a realização de curativos, a aspiração de vias aéreas, a intubação endotraqueal, retiradas de drenos, dentre outros, o que pode gerar desconforto, estresse e dor (Cardoso et al., 2015).

Objetivo deste estudo é relatar um caso de malformação neonatal de três tipos: dedo extranumerário em mão esquerda, genitália ambígua e anus impérvio.

\section{Metodologia}

O presente estudo trata-se de um relato de caso, com informações obtidas por meio de revisão de ase documental do tipo prontuário. Este leva em consideração a resolução n ${ }^{\circ}$ 510, de 07 de abril de 2016 do Conselho Nacional de Saúde, que justifica a dispensa de TCLE para pesquisas que objetivam o aprofundamento teórico de situações que emergem espontânea e eventualmente na pratica profissional, desde que não seja revelado dados que possam identificar de alguma forma o sujeito pesquisado (Conselho Municipal De Saúde, 2016).

Atrelado a isto, cabe ressaltar para que tivessem acesso ao prontuário do paciente, as pesquisadoras primeiramente precisaram da autorização da instituição, que possibilitou parecer positivo. Diante disso, afim de manter o respeito pela pesquisa científica, foi assinado o Termo de Compromisso de Utilização de Dados (TCUD) pela pessoa que entraria em contato com o prontuário, a pesquisadora responsável.

Esta pesquisa, a partir deste momento, divide-se em duas etapas. A primeira diz respeito ao relato de caso, que consiste em uma análise profunda e exaustiva de maneira que permita um amplo e detalhado conhecimento sobre o estudo (Gil, 2008). Além disso, levanta pontos principais dentro do contexto do quadro clinico do paciente que favoreçam o entendimento sobre a sua condição e fornece subsídios fundamentais para o melhor tratamento deste em determinadas situações (Yoshida, 2007).

A segunda se configura em parte essencial do relato de caso, diz respeito a busca da literatura, traçada com o objetivo de aprofundar o conhecimento no universo estabelecido pelas condições do caso clinico do paciente. Destarte, esta pode ser 
caracterizada como uma estratégia reprodutível explícita para o rastreio e inclusão de estudos, análise apropriada e apresentação dos resultados, interpretações apoiadas por dados, implicações para futuras pesquisas e se for o caso, para a política ou prática (Ravindran Et Shankar, 2015).

\section{Relato de Caso}

Recém-nascido pré-termo (RNPT), de 35 semanas, Pequeno para Idade Gestacional (PIG), sexo indeterminado, nascido de parto cesáreo em município de Tucuruí-PA, DN: 19/09/2019. Apgar 7/9, Peso de nascimento (PN) 1.410g, PC: 31 cm, PT: 26 cm. Mãe 28 anos, G1 P0 A0, realizou pré-natal, sorologias negativas, com Diagnostico de SHEG, nega doenças crônicas e/ou infecto contagiosas. RN deu entrada na Unidade de Terapia Intensiva Neonatal (UTIN) procedente do centro cirúrgico nascido de parto de urgência por provável DPP, mãe com SHEG, chorou ao nascer, necessitou de VPP com ambu e máscara com boa recuperação, evoluiu com DR, diagnóstico de Malformações tipo: Dedo extranumerário em mão esquerda, Genitália Ambígua e Anus impérvio.

Ao exame físico apresentava-se em Regular Estado Geral (REG), reativo, hidratado, crânio simétrico, fontanelas normotensas, edema palpebral, Acrocianose, taquipnéico, Tiragem subcostal. Tórax simétrico, eupnéico, AP: MV+ sem RA, AC: BCNF $2 \mathrm{~T}$ s/s. Abdômen globoso e flácido, RHA +. Genitália ambígua com presença de testículo em provável bolsa escrotal. Presença do canal anal, e anus impérvio. Funções fisiológicas diurese presente e evacuações ausentes.

Condutas: Instalação de CPAP nasal, cateter umbilical (CUMB), Dieta Zero, SOG nº 8 aberta, Raio X de tórax e abdômen, USG de abdômen exames laboratoriais (hemocultura, ionograma, PCR, TS, VDRL, Cariótipo G Banda), introdução de Antibióticoterapia e solicitação de avaliação e conduta da Cirurgia Pediátrica. No $1^{\circ}$ Dia Internação Hospitalar (DIH) ativo e reativo, hidratado, confortável sob CPAP nasal porem não tolera retirada com algumas apneia, introduzido ATB, avaliado pela Cirurgia pediátrica e solicitado TFD, passado SOG n ${ }^{\circ} 10$ devido distensão abdominal. $2^{\circ}$ dia internação RN submetido a procedimento cirúrgico de ileostomia com inserção de bolsa de colostomia, retirado CUMB, foi intubado devido procedimento e encaminhado novamente para POI em unidade neonatal. $3^{\circ}$ dia de internação RN apresentou cianose sem entrada de ar nos pulmões retirado TOT, presença de rolha, recuperou-se bem após extubação acomodado em VNI. $4^{\circ}$ dia de internação retirado CPAP nasal e realizado dissecção de veia para continuidade de tratamento medicamentoso, ativo e reativo, em ar ambiente confortável Spo2 97\%, FC: 148 bpm, SOG com RG salivar, colostomia funcionante com baixo debito, o mesmo saiu de T, FD para continuação de tratamento devido hospital não possuir recurso para seu tratamento.

\section{Discussão}

Para Pereira et al., (2011) a chegada de um filho é um momento de muitas expectativas geradas por parte da família, entretanto, o choque em receber um filho com a presença de alguma anomalia pode representar uma grande carga emocional e social na vida dos pais, visto que essas crianças, quando não forem vão ao óbito no primeiro mês de vida ou ao nascer, precisarão de maior atenção e cuidado, essa questão torna-se ainda mais relevante em termos jurídicos, pois, as gestações de fetos malformados no Brasil são levadas a termo, salvo raras exceções, em que são apresentadas as malformações incompatíveis com a vida, onde as liminares judiciais são obtidas por casais que optam pela interrupção da gestação.

Acredita-se que conhecer o perfil das crianças nascidas com malformação congênita seja importante para a assistência, no sentido de oferecer subsídios à melhoria da qualidade da demanda profissional prestada no nascimento de uma criança com malformação congênita (Rodrigues et al., 2014).

Assim, várias anormalidades podem ser ainda observadas durante a gestação, na fase neonatal, ou mesmo em determinado período após a concepção, que ocasionam danos constantes e irreversíveis, portanto, o acompanhamento do desenvolvimento fetal via ultrassonografia que deve ser parte da rotina das consultas do pré-natal, permite assegurar para os 
pais o bem-estar gestacional, dessa forma, é necessário que os exames ultrassonográficos sejam realizados em vários momentos da gestação, pois, através dessa visualização é possível detectar vários diagnósticos de acordo com o período da manifestação dos primeiros sinais (Lima et al., 2018).

As anomalias congênitas está associada ao maior índice à morbimortalidade infantil, principalmente no período neonatal, tornando-se importante o seu diagnóstico precoce para o planejamento e a alocação de recursos dos serviços de saúde especializados (pré-natal, natal e pós-natal), para a redução da morbimortalidade, principalmente neonatal precoce, e para a melhora da qualidade de vida e dos índices de sobrevida (Cosme, Lima \& Barbosa, 2017).

Para Campos et al., (2017) a alta prevalência das malformações congênitas está associada à deficiência de informações e do desconhecimento de estratégias preventivas, a isto, se somam "a ausência de políticas públicas de saúde eficazes, que envolvam contracepção, imunização contra doenças infectocontagiosas maternas, planejamento familiar, cuidados pré-concepcionais, suplementação vitamínica e realização de diagnóstico pré-natal” (p.2).

Para diminuir as taxas de morbimortalidade no período neonatal, torna-se necessário conhecer as particularidades regionais de cada serviço de UTIN, como a frequência das malformações congênitas, a identificação dos fatores de riscos associados, além de valorizar o diagnóstico precoce e faça medidas de prevenção primária e secundária (Cristovam, et al. 2019).

Logo, vale ressaltar que a existência de algum tipo de malformação congênita tanto no nascimento, como na causa do óbito de crianças é atrelado ao sub-registro, ou seja, ainda existem falhas ao coletar informações acerca deste tipo de ocorrência observada em todas as regiões do Brasil (Centro-Oeste, Nordeste, Norte, Sul e Sudeste), o que revela a importância de se trabalhar mais fortemente as políticas de promoção e prevenção, direcionada a saúde materno e infantil, para que sejam minimizados o acontecimento de malformações (Santos, 2018).

Os sub-registros dessas informações são identificados em todas as regiões do país, considerando o contexto e particularidade de cada uma. Uma vez que essas regiões têm preenchimentos inadequados das declarações e consequentemente na alimentação dos sistemas, acabam dificultando o monitoramento de determinadas malformações que acometem determinada população ou que se encontra em situação de risco (ibidem, 2018, p.11).

Sabemos que a inserção de sonda gástrica no recém-nascido internado na UTIN é um dos procedimentos de enfermagem mais corriqueiros e que está indicada para a alimentação, para a administração de medicamentos e para a descompressão gástrica (Brasil, 2019).

As condutas frente a este caso foi a passagem da sonda orogástrica SOG) $n^{\circ} 10$ para instalação do sistema de Venturi para aspiração da Vias Aéreas Superiores (VAS), devido produção excessiva e para evitar de utilizar sistema ar comprimido para aspiração além de evitar trauma evita ruídos desnecessário.

Como o recém-nascido foi submetido a procedimento cirúrgico a equipe de enfermagem tem que aplicar a escala de dor a que foi utilizada foi a de NIPS, sendo que acima de 3 pontos tem que avaliar para condutas não farmacológica para depois se utilizar a farmacológica conforme prescrição medica ou dependendo da situação irá se utilizar ambas em conjunto, para amenizar tudo que possa ser agressor ao neonato.

Dessa forma, a avaliação da dor no período neonatal é indispensável para os recém-nascidos criticamente doentes e que são submetidos a diversos procedimentos invasivos, essa avaliação baseia-se nas respostas obtidas do recém-nascido de acordo com estímulo nociceptivo que é recebido, ou seja, o modelo de avaliação da dor no neonato é verificado através das modificações de órgãos, sistemas e comportamentos ocorridos após um evento doloroso provocado, ademais, o neonato possui uma maneira característica e própria em responder à dor, e o reconhecimento da transmissão desta linguagem por parte do 
profissional que o assiste é fundamental para uma avaliação adequada do fenômeno nociceptivo e para o emprego de um tratamento eficaz e adequado (Balda \& Guinsburg, 2018).

As principais variáveis comportamentais verificadas no neonato no contexto da dor são: choro, atividade motora e mímica facial de dor. Estas respostas refletem a experiência dolorosa após o estímulo nociceptivo, incluindo os aspectos sensoriais e emocionais intrínsecos à dor, além de diferenças individuais na expressão dessas respostas (ibidem, 2018, p.3).

A equipe de enfermagem frente a todos os neonatos tem papel fundamental pois prestam atendimento durante as 24 horas dentro da UTIN, o enfermeiro tem sua função em avaliar este recém-nascidos para tomar condutas imediatas, evitando assim atraso nos procedimentos juntamente com o técnico de enfermagem, o recém-nascido foi logo intubado onde a equipe auxilia neste procedimento de sedação e acomodação do mesmo no leito e monitorização (Brasil, 2011).

Sendo assim, o enfermeiro tem sua atuação além do âmbito hospitalar, pois, este possui papel fundamental detectar precocemente qualquer anormalidade através da realização de triagem e rastreamento dessas patologias por meio de consultas de enfermagem durante o pré-natal, além de esclarecer e fornecer informações acerca do quadro da malformação, ofertando segurança e confiança, evitando assim, interpretações erradas, desencontros e paralisações nas relações tanto da equipe quanto da família, sendo portanto, um facilitador na formação do vínculo mãe e filho com anomalias congênitas (Brito et al., 2019).

O neonato necessitou ser transferido para tratamento fora domicilio (TFD) devido à complexidade da malformação necessitando de outros exames e procedimentos cirúrgicos e o hospital não possui este tipo de especialidade, a Portaria SAS n ${ }^{\circ}$ 055, de 24 de fevereiro de 1999, que dispõe sobre o Tratamento Fora de Domicílio tem por objetivo garantir o acesso de pacientes de um município a serviços assistenciais em outro município, ou ainda, em caso especiais, de um Estado para outro Estado, além de ajudar na garantia de transporte para tratamento e hospedagem, quando necessário. O TFD contempla exclusivamente, os pacientes atendidos na rede pública e referenciada, reforçando a necessidade de se obter dados que demonstrem a realidade de cada local para proporcionar recursos necessários na ocorrência de tais agravos e assim facilitar o acesso ao tratamento e recursos terapêuticos mais eficazes, contribuindo para deslocamento desnecessário à outros municípios em busca dos centros de referência (Brasil, 1999).

\section{Considerações Finais}

O presente estudo teve como objetivo avaliar um relato de caso de um recém-nascido com três tipos de malformações congênita em uma unidade neonatal, que na maioria das vezes estes hospitais não possuem recurso para tratar tais situações necessitando diversas vezes referenciar a outra unidade para realizar tratamento fora do domicilio (TFD).

Dessa forma, a contribuição deste estudo constitui grande relevância para o norteamento na adoção de medidas para o fortalecimento da política do pré-natal com condutas preventivas e delineamento de ações estratégicas no combate aos fatores e causas evitáveis afim de reduzir essas alterações que podem trazer sequelas irreversíveis aos neonatos no seu processo de crescimento e desenvolvimento.

Portanto, se faz necessário mais estudos com levantamento de dados e perfis epidemiológicos nas unidades de terapia intensiva com neonatos apresentando malformação congênita e correlacionar com os achados clínicos obstétricos da mãe e da criança, pois, esta é uma condição de grande importância para a saúde pública, visto que, se tornou um problema social no mundo desafiando os profissionais de saúde e a comunidade cientifica, 


\section{Referências}

Amorim, M. M. R. D., Vilela, P. C., Santos, A. R. V. D., Lima, A. L. M. V., Melo, E. F. P. D., Bernardes, H. F., ... \& Guimarães, V. B. (2006). Impacto das malformações congênitas na mortalidade perinatal e neonatal em uma maternidade-escola do Recife. Revista Brasileira de Saúde Materno Infantil, 6, s19-s25.

Associação Dos Deficientes Físicos Do Paraná (ADFP) (2021). Malformação Congênita: o que é $e$ como prevenir, detectar e tratar? http://adfp.org.br/blog/2021/03/02/malformacao-congenita-o-que-e-e-como-prevenir-detectar-e-tratar/.

Balda, R. D. C. X., \& Guinsburg, R. A linguagem da dor no recém-nascido Atualizado em dezembro de 2018.

Biblioteca Virtual Em Saúde (BVS) (2021). Ministério da saúde. “Muitos defeitos de nascença, uma voz”: 03/3 - Dia Mundial dos Defeitos do Nascimento. http://bvsms.saude.gov.br/component/content/article?id=3432.

Brasil (2018). Ministério da Saúde Secretaria de Ciência, Tecnologia e Insumos Estratégicos. Departamento de Ciência e Tecnologia. Agenda de Prioridades de Pesquisa do Ministério da Saúde - APPMS [recurso eletrônico] / Ministério da Saúde, Secretaria de Ciência, Tecnologia e Insumos Estratégicos, Departamento de Ciência e Tecnologia. - Brasília: Ministério da Saúde, 2018. 26 p. : il.

Brasil (2019). Ministério da saúde. Instituto Fernandes Figueira. Portal de Boas Práticas. Atenção ao recém-nascido. Principais Questões sobre Cuidados com o Recém-nascido na UTI Neonatal. Setembro, 2019. https://portaldeboaspraticas.iff.fiocruz.br/atencao-recem-nascido/principais-questoes-sobre-cuidadoscom-o-recem-nascido-na-uti-neonatal.

Brasil (2011). Ministério da Saúde. Secretaria de Atenção à Saúde. Departamento de Ações Programáticas e Estratégicas. Atenção à saúde do recém-nascido: guia para os profissionais de saúde / Ministério da Saúde, Secretaria de Atenção à Saúde, Departamento de Ações Programáticas e Estratégicas. - Brasília: Ministério da Saúde, 2011.

Brasil (1999). Portaria $n^{\circ}$ 55, de 24 de fevereiro de 1999. Dispõe sobre a rotina do tratamento fora de domicilio no Sistema único de saúde - sus, com inclusão dos procedimentos específicos na tabela de procedimentos do sistema de informações ambulatoriais do sia/sus e dá outras providências. Https://bvsms.saude.gov.br/bvs/saudelegis/sas/1999/prt0055_24_02_1999.html.

Brito, A. P. M., Ribeiro, K. R. A., De Paula Duarte, V. G., \& De Abreu, E. P. (2018). Enfermagem No Contexto Familiar Na Prevenção De Anomalias Congênitas: Revisão Integrativa. Journal Of Health \& Biological Sciences, 7(1 (JAN-MAR)), 64-74.

Campos, D. A., Melo, M. D. S. R., De Souza Medeiros, B. G., \& Lucena, V. S. (2018). Fatores genéticos: prevalência de mortalidade neonatal e anomalias congênitas. Journal of Biology \& Pharmacy and Agricultural Management, 13(2).

Cardoso, M. V. L. M. L., Lima, V. R. M., Fontoura, F. C., Rodrigues, S. E., Saraiva, I. A., \& Fontenele, F. C. (2015). Terapêuticas utilizadas em recémnascidos com malformações congênitas internados em unidade neonatal. Revista Eletrônica de Enfermagem, 17(1), 60-8.

Cristovam, M. A., Dallago, R. T., Fiori, C. M. M. C., Sandrini, F., De Moraes, M., Rover, S., ... \& Bresolin, A. C (2019). Frequência de anomalias congênitas em uma Unidade de Terapia Intensiva Neonatal no Brasil.

Conselho Municipal De Saúde (2016). Resolução $n^{\circ}$ 510, de 07 de abril de 2016. Dispõe sobre as normas aplicáveis a pesquisas em Ciências Humanas e Sociais cujos procedimentos metodológicos envolvam a utilização de dados diretamente obtidos com os participantes ou de informações identificáveis ou que possam acarretar riscos maiores do que os existentes na vida cotidiana, na forma definida nesta Resolução. Http://conselho.saude.gov.br/resolucoes/2016/Reso510.pdf.

Cosme, H. W., Lima, L. S., \& Barbosa, L. G. (2017). Prevalência de anomalias congênitas e fatores associados em recém-nascidos do município de São Paulo no período de 2010 a 2014. Revista Paulista de Pediatria, 35(1), 33-38.

Gil, A.C. Como elaborar projetos de pesquisa. 4. ed. São Paulo: Atlas, 2008.

Lima, M. P., Santos, J. A. M., Rockembach, J. A., Dos Santos Lochmis, F., \& De Souza Lima, B. S. (2019). Perfil De Malformação Congênita Em Nascidos Vivos, Do Estado Do Rio Grande Do Sul, 2012-2016. Revista De Saúde Dom Alberto, 3(1), 105-120.

Pereira, R. J., De Abreu, L. C., Valenti, V. E., Albuquerque, W. D. M., Pereira, S. C., Araújo, R., ... \& Dos Santos, S. M. (2008). Freqüência de malformações congênitas das extremidades em recém-nascidos. Journal of Human Growth and Development, 18(2), 155-162.

Pereira, P. K., Lima, L. A., Legay, L. F., Santos, J. F. D. C., \& Lovisi, G. M. (2011). Malformação congênita do bebê e risco de transtornos mentais maternos durante o período gravídico-puerperal: uma revisão sistemática. Caderno Saúde Coletiva, 19(1), 2-10.

Ravindran, V., Shankar, S (2015). Systematic reviews and meta-analysis demystified. Indian J. Rheumatol. 10, 89-94. 2015.

Rodrigues, L. D. S., Lima, R. H. D. S., Costa, L. C., \& Batista, R. F. L. (2014). Características das crianças nascidas com malformações congênitas no município de São Luís, Maranhão, 2002-2011. Epidemiologia e Serviços de Saúde, 23, 295-304.

Reis, L. D. L. A. S. D., \& Ferrari, R. (2014). Malformações Congònitas: Perfil Sociodemográfico Das Mçes E Condições De Gestação. Journal of Nursing UFPE/Revista de Enfermagem UFPE, 8(1).

Santos, E. M. D. (2018). Malformação congênita no Brasil: uma análise dos nascimentos e óbitos infantis no período de 2001 a 2015 (Bachelor's thesis).

Yoshida, W. B (2007). Redação Do Relato De Caso. J Vasc Bras. 6(2), 112-113. Https://www.scielo.br/pdf/jvb/v6n2/v6n2a04.pdf. 